\title{
A multicore compound glass optical fiber for neutron imaging
}

\author{
Michael Moore $^{\mathrm{a}, *}$, Xian Feng ${ }^{\mathrm{b}}$, Gilberto Brambilla ${ }^{\mathrm{b}}$, Jason Hayward ${ }^{\mathrm{a}}$ \\ ${ }^{a}$ Department of Nuclear Engineering, University of Tennessee, Knoxville, TN 37996-2300, USA \\ ${ }^{\mathrm{b}}$ Optoelectronics Research Centre, University of Southampton, Southampton, SO17 1BJ, UK
}

\begin{abstract}
Optical fibers have been successfully utilized for point sensors targeting physical quantities (stress, strain, rotation, acceleration), chemical compounds (humidity, oil, nitrates, alcohols, DNA) or radiation fields (X-rays, $\beta$ particles, $\gamma$ rays). Similarly, bundles of fibers have been extremely successful in imaging visible wavelengths for medical endoscopy and industrial boroscopy. This work presents the progress in the fabrication and experimental evaluation of multicore fiber as neutron scattering instrumentation designed to detect and image neutrons with micron level spatial resolution.
\end{abstract}

Keywords: High Spatial Resolution, Multicore, Neutron Imaging, Optical Fiber, Scintillators

\section{INTRODUCTION}

Optical fibers have found numerous applications in distributed and point sensors aimed at measuring physical measurables (most notably strain, temperature, rotation and acceleration), chemical compounds (ranging from humidity to explosives, combustibles and biological compounds like proteins or DNA) or radiation fields (X-rays, $\beta$ particles, $\gamma$ rays). As far as the monitoring of heavy charged particles and high energy photons is concerned, detection is often achieved by exploiting scintillation. An incident radiation field enters a glass matrix and generates highly energetic charged particles that dissipate their kinetic energy into the ionization of the matrix atoms. The dissipated energy may be partly transferred to a dopant (typically rare earths, like $\mathrm{Ce}$ ), which emits scintillation light in the visible/near UV when de-exciting. Scintillation light can then detected and related to the incident radiation by conventional photomultipliers or charged coupled devices $(\mathrm{CCDs})^{1-3}$.

Scintillating glasses have been introduced into fiber cores to provide remote sensing capabilities ${ }^{4}$ for a variety of high energy fields including, X-rays, $\gamma$-rays and charged particles ${ }^{5}$ like protons or $\beta$ particles. Neutrons represent more of a challenge, as they do not possess charge. Their detection often relies on nuclear absorption reactions that produce charged particles that can then be detected through conventional scintillation processes ${ }^{6}$.

In our work, neutrons are detected by exploiting their well-known reaction with ${ }^{6} \mathrm{Li}$ (see Fig. 1), which generates two charged particles ( $\alpha$ and tritium), which can then interact with the scintillating medium. The alpha and tritium particles have a total combined kinetic energy of $4.8 \mathrm{MeV}$ which excites the $\mathrm{Ce}^{3+}$ atoms in the glass, which in turn emit light in the 395-432 nm wavelength range. The absorption cross section for ${ }^{6} \mathrm{Li}$ is dependent on the neutron energy, with the highest probabilities at cold/thermal energies.

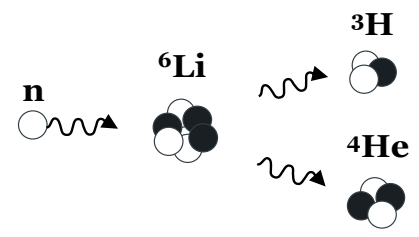

Figure 1. Schematic of the reaction between a neutron (n) and a ${ }^{6} \mathrm{Li}$ atom, producing tritium $\left({ }^{3} \mathrm{H}\right)$ and an $\alpha$ particle $\left({ }^{4} \mathrm{He}\right)$.

Bundles of fibers have been extremely successful in imaging visible wavelengths for medical endoscopy and industrial boroscopy, but the relatively low density of fibers has produced limited neutron imaging capabilities ${ }^{7}$. While high resolution X-ray imaging is able to resolve features at the nanoscale, the state-of-the-art for neutron imaging research is $\sim 15 \mu \mathrm{m}^{8}$. Here we present the progress in the fabrication of multicore fiber designed to enable high spatial resolution for neutron imaging applications in science and engineering.

*mmoor138@utk.edu; 


\section{OPTICAL FIBER FABRICATION}

The multi-core optical fiber was fabricated through stacking unit elements composed by Guardian Glass (Nucsafe Inc., Oak Ridge, TN, USA), a scintillating glass, as core and a silicate glass as cladding. The core was doped with isotopically enriched ${ }^{6} \mathrm{Li}$ for the detection of neutrons and $\mathrm{Ce}^{3+}$ for scintillation. The cladding had a composition similar to the LLF1 glass (Schott AG, Mainz, Germany). The two glasses were chosen because of their similar thermal properties that allow for co-drawing. The core glass, provided in a cylindrical rod with a diameter of $7.5 \mathrm{~mm}$, was inserted into a cladding tube with an external square cross section. The set of core/cladding rod-in-tube was caned to an external diameter of $730 \mu \mathrm{m}$ (Fig. 2(a)) at which the core had a diameter of $460 \mu \mathrm{m}$. The square cane was then stacked into a square array illustrated in Fig. 2(b). The fused array was consolidated in a furnace to produce a monolithic square preform (Fig. 2(c)) before being drawn into the fiber. The fiber had a width ranging from $126 \mu \mathrm{m}$ to $160 \mu \mathrm{m}$

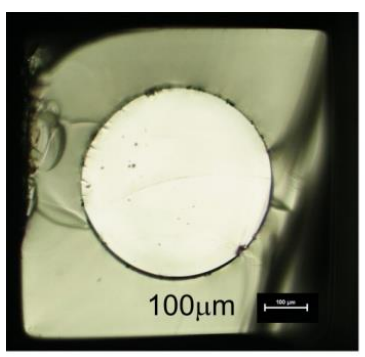

(a)

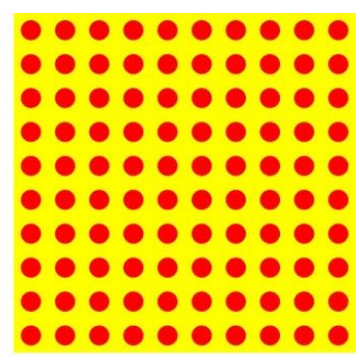

(b)

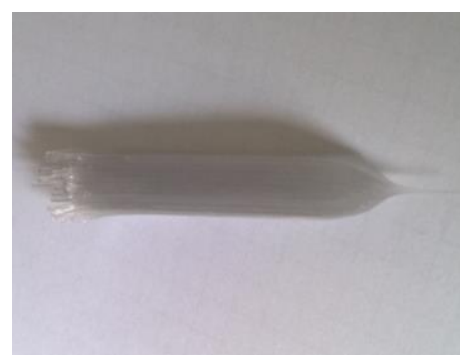

(c)

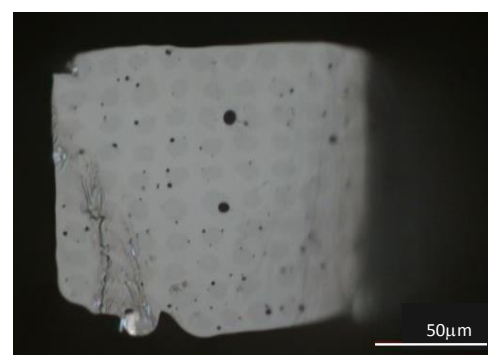

(d)

Figure 2. (a) Optical photo of elongated core/cladding square cane. The side length of the cane is $730 \mu \mathrm{m}$ and the core diameter is $460 \mu \mathrm{m}$. (b) Schematic of stack of 10x10 canes before final draw. (c) Photograph of the stack. On the right it is possible to see the neckdown region where the preform is pulled into a fiber. (d) Optical photo of ${ }^{6} \mathrm{Li}$ glass multicore fiber. The fiber width is $140 \mu \mathrm{m}$, while the core diameter is $7 \mu \mathrm{m}$.

Fig. 2(d) shows a cross section of the final multicore fiber, which presents a number of microbubbles, resulting from the cavities left in the preform during consolidation. As already observed in other scintillating fibers, the presence of small bubbles is not believed to be detrimental to the fiber operation in that it increases the numerical aperture the fiber cores, thus decreasing the core cross talk. However, uniformity is required for ideal high resolution neutron imaging.

Since a larger field of view was desired with which to detect neutrons, an array of scintillating fibers was produced. In order to create an array of the fiber, it was loaded onto a glass plate with a rigid UV curable epoxy for further processing. The glass plate was then cut into two pieces, stacked together, and the top glass plate ground away. After re-assembly, the new two layer array was then cut in half and re-assembled to produce a four layer array. This process was repeated to create a 64 by 64 array of the fibers with a thickness of $1.7 \mathrm{~mm}$. The array was finished by polishing the surface, cutting the array from the glass, and leaving glass sidewalls in place for support.

\section{TESTING WITH NEUTRON SOURCES}

To determine how the drawing process has affected the scintillation performance of the Guardian Glass core and to verify that scintillation light is being efficiently channeled with micron-level architecture, the fiber array was assayed with a cold neutron source. Testing was carried out at the High Flux Isotope Reactor's CG-1D beamline at Oak Ridge National Laboratory, Oak Ridge TN, USA. Initially, a nominal background was observed when a calibrated Hamamatsu photomultiplier tube (PMT) R977 was placed in the beamline cage without the fiber array. The entire surface of the fiber array was then secured to the PMT window, wrapping it first with Teflon and then black tape. The response of the fiber array to the background (neutron shutter closed), and to the neutron beam (neutron shutter open) was observed. Scintillation was clearly seen for both cases (see Fig. 3). The response to the neutron beam displays an increased integrated charge distribution. Thus, cold neutrons clearly exhibit a higher light output response than the gamma background radiation present in the beamline.

It was found that the PMT had an integrated charge response to a single photon of $122 \pm 60 \mathrm{fC}$ under a bias of $-1,500 \mathrm{~V}$. The neutron response yields an approximate photoelectron number of 246 photoelectrons. Previous analysis of a larger monolithic Guardian Glass sample with dimensions of $23.88 \mathrm{~mm}$ (diameter) by $2.85 \mathrm{~mm}$ (thickness) established that 
Guardian Glass can be expected to produce $\sim 9,000$ photoelectrons per thermal neutron capture ${ }^{8}$. The difference in light output between the two experiments is a result of the difference in scintillator form-monolithic crystal vs. thin fibersand the small surface area of the scintillator in contact with the PMT window. The Guardian Glass cores in the fiber array had an approximate surface area of $15.7 \mathrm{~mm}^{2}$, compared to the bulk sample's $447.9 \mathrm{~mm}^{2}$.

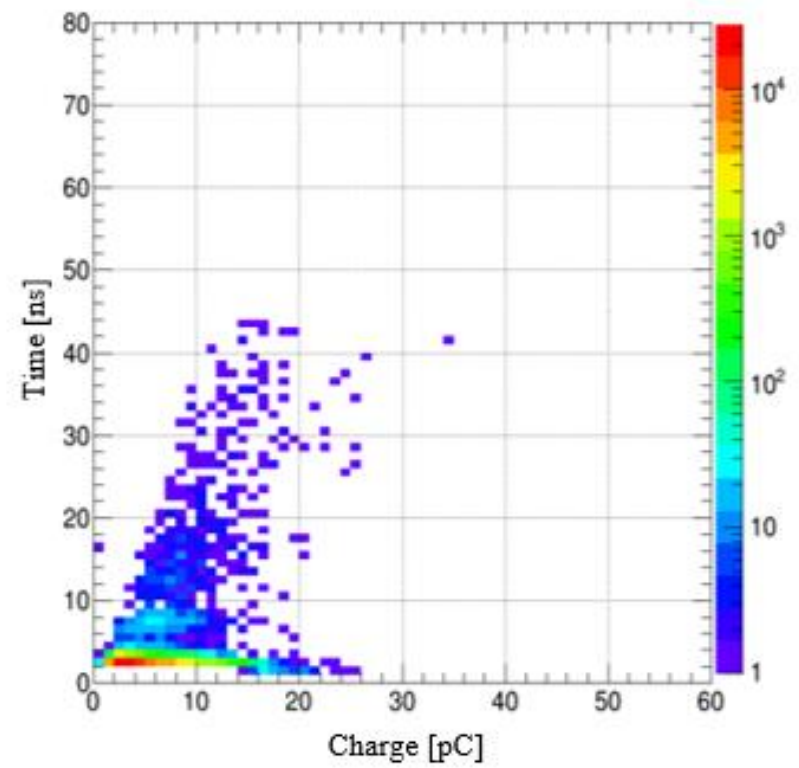

(a)

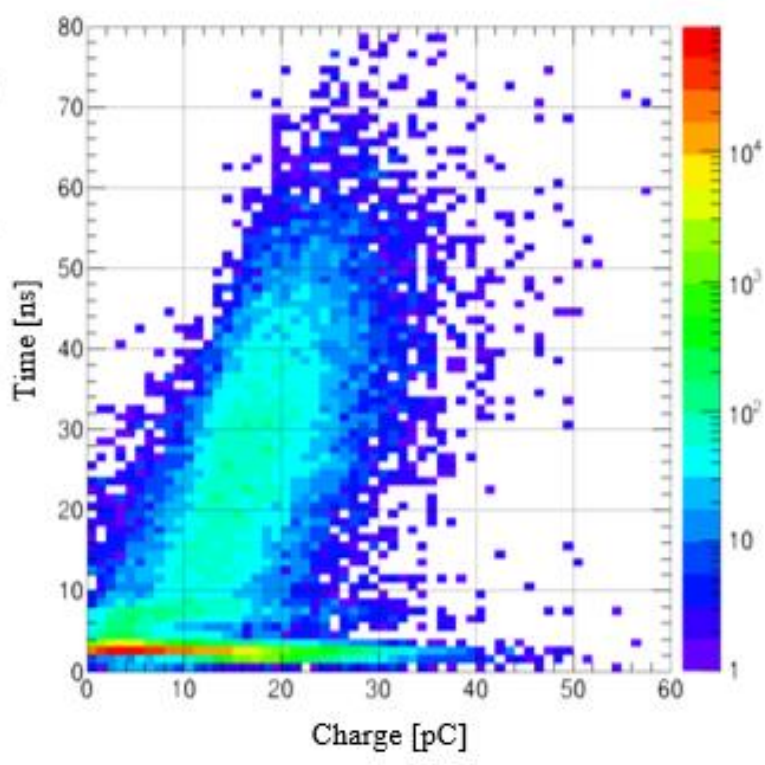

(b)

Fig. 3. PMT response to the presence of scintillation in the fiber array at CG-1D to (a) background radiation, in the beamline, and (b) the neutron beam.

After the PMT measurements confirmed the presence scintillation, a SBIG CCD STF-8300M with a Canon MP-E 65 $\mathrm{mm} \mathrm{f} / 2.81-5 \mathrm{x}$ lens was focused to the mirrored fiber array. Since the emission wavelength of $\mathrm{Ce}^{3+}$ is in the $395-432 \mathrm{~nm}$ wavelength range, focusing was accomplished using blue light, which has a 450-495 nm wavelength range. From the blue light image, an absolute system resolution was obtained by measuring the line spread function. By analyzing the epoxy bond gaps of the glass plating in several locations, it was determined that the ultimate resolution of the setup was approximately $10 \mu \mathrm{m}$ at $8 \%$ contrast.

Without changing the focus of the system, 91 images with $600 \mathrm{~s}$ exposure times were taken in the neutron beam. A cadmium-coated edge was placed in the path of the neutron beam to observe the edge response (see Fig. 4). These images were processed so bright outliers of 50 gray count and above are replaced by the median of the pixels in the surrounding area for each image to remove cosmics and gamma-rays that interacted directly with the Multi-Channel Plate (MCP) and the CCD device itself. Then all runs were stacked with a median filter which again reduces noise in the active image by replacing each pixel with the median of the neighboring pixel values. Twelve images with $600 \mathrm{~s}$ exposure times were taken with the system with no neutron beam (shutter closed) for a total of 2 hours. This background data was handled with a median filter after removing outliers as done previously, and normalized to $600 \mathrm{~s}$ of beam time. The background image was subtracted from the neutron beam image to produce an image of the neutron light without inherent bias and dark noise from the CCD. While the response to the cadmium coated edge does further demonstrate the sensitivity of the fiber array to cold neutrons, unfortunately, the edge was not thick enough or close enough to the fiber array to allow for a resolution measurement of the scintillation light.

\section{FUTURE WORK AND CONCLUSIONS}

Using lessons learned from the measurements at CG-1D, we are working on a new design and improved characterization setup. We are creating a new generation of microfiber with improved packing fraction of cores, 40\%, and a higher NA value, 0.44 . Additionally, we are characterizing an Intensified CCD coupled to a UV sensitive optical system, stationed 
on a negative stiffness vibration isolator, capable of resolving $\mu \mathrm{m}$ features. At $10 \%$ of the Modulation Transfer Function (MTF), the resolvable spatial frequency is $\sim 3.3$ cycles $/ \mu \mathrm{m}$ with blue light.

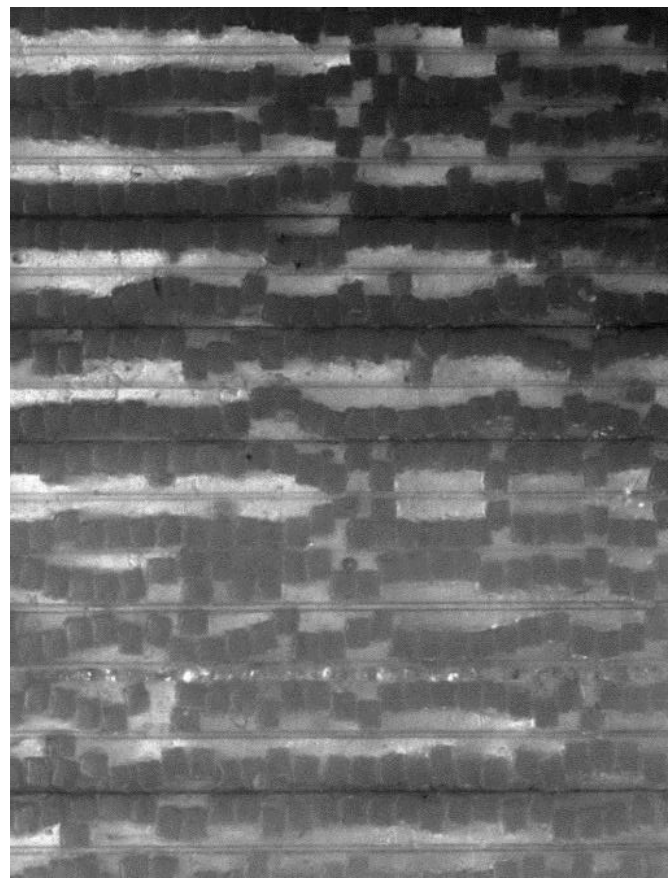

(a)

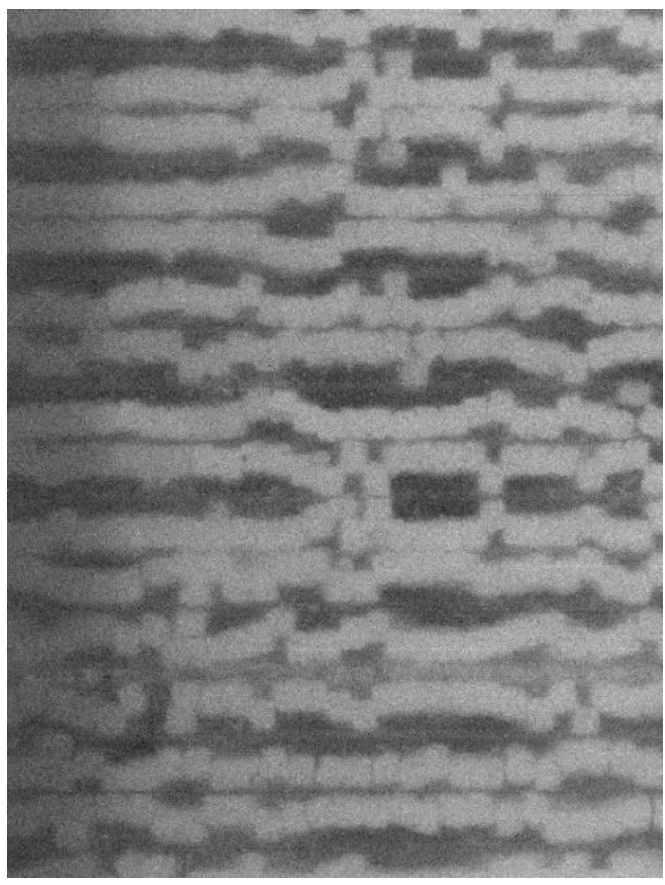

(b)

Fig. 4. (a) Blue light image of the scintillating fiber array with $10 \mu \mathrm{m}$ resolution, (b) Processed scintillation light image of the array normalized to an exposure time of $600 \mathrm{~s}$. Less scintillation light can be seen on the left side of the image. This shadow was caused by the attenuation of the cold neutrons by the cadmium coated edge.

\section{REFERENCES}

[1] Nikl, M. "Scintillation detectors for x-rays," Meas. Sci. Technol. 17, R37-R54 (2006).

[2] Leutz, H. "Scintillating fibres," in Nuclear Instruments and Methods in Physics Research Section A Accelerators Spectrometers Detectors and Associated Equipment 364 (3), 422-448 (1995).

[3] Miyanaga, N., Ohba, N., and Fujimoto, K. "Fiber scintillator/streak camera detector for burn history measurement in inertial confinement fusion experiment," Review of Scientific Instruments. 68 (1), $621-623$ (1997).

[4] Vedda, A., Chiodini, N., Di Martino, D., et al. "Ce3+ -doped fibres for remote radiation dosimetry," Applied Physics Letters, 85 (26), 6356-6358, (2004).

[5] Chiodini, N., Vedda, A., Fasoli, M., et al. "Ce doped $\mathrm{SiO} 2$ optical fibers for remote radiation sensing and measurement," in, Udd, Eric, Du, Henry H. and Wang, Anbo (eds.) Fiber Optic Sensors and Applications VI. SPIE Defense and Security Bellingham, USA, Society of Photo-Optical Instrumentation Engineers, 7316 (2009).

[6] Ottonello, P., Rottigni, G.A., Zanella, G., and Zannoni, R. "Neutron Imaging with Scintillating-Glass FiberOptic Plates," Journal of Neutron Research 4(1-4), 109-116 (1996).

[7] Buckles, R.A., Ali, Z.A., Cradick, J.R., et al. "Scintillating Fiber Array Characterization and Alignment for Neutron Imaging using the High Energy X-ray (HEX) Facility,” in Proc. The Sixth International Conference on Inertial Fusion Sciences and Applications (2009).

[8] Williams, S. H., et al. "Detection system for microimaging with neutrons," Journal of Instrumentation 7.02 (2012): P02014.

[9] Song, Y., Conner, J., Zhang, X., Hayward, J. "Monte carlo simulation of a very high resolution thermal neutron detector composed of glass scintillator microfibers," Appl. Radiat. Isot. 108 (2015) 100-107. 